\title{
FERROMAGNETISM IN HUBBARD CHAINS
}

\author{
M. Cuoco, C. Noce and A. Romano \\ Unità I.N.F.M. di Salerno - Dipartimento di Fisica Teorica e S.M.S.A. \\ Università di Salerno, 84081 Baronissi (Salerno), Italy
}

We analyze the possible occurrence of ferromagnetism in the Hubbard model, by means of an exact diagonalization study performed on 3- to 8-site chains with periodic boundary conditions. In the case of one hole in the half-filled configuration, we find that the Nagaoka state is reached only in the 3 - and in the 4-site case. Ground states characterized by unsaturated ferromagnetism are found when the case of more than one hole is considered.

PACS numbers: 71.10.Fd, 75.10.Lp

\section{Introduction}

The problem of ferromagnetism in systems of itinerant electrons is a topic of great current interest in condensed matter physics. One of the theoretical models considered as a good starting point to investigate this intriguing problem is certainly the Hubbard model.

In the framework of this model some rigorous examples of ferromagnetism have been obtained for special choices of the lattice and of the electron filling. Only for one-dimensional systems with open boundary conditions the situation is relatively simple: indeed, the theorem of Lieb and Mattis [1] ensures that the ground state is never ferromagnetic. In presence of a hard-core repulsion between electrons, if the dynamically allowed permutations are all even, there is one among the ground states that has a maximal spin $S$ equal to half of the number of electrons (saturated ferromagnetism) [2]. Besides, if the lattice obeys a certain connectivity condition [3] (which is not satisfied for example in the one-dimensional case for a number of sites greater than 4) and there is exactly one hole, the ferromagnetic ground state is unique apart from the degeneracy due to the global $S U(2)$ invariance of the Hubbard Hamiltonian. This is the well-known Nagaoka theorem.

A unique ground state that may have a macroscopic but not saturated value for the spin is also obtained for the Hubbard model on a bipartite lattice if the number of electrons is equal to the number of sites. This result is contained in the theorem of Lieb [4], which provided the first example of itinerant-electron ferromagnetism under no assumption of infinite on-site repulsion.

Another rigorous example is represented by the flat-band ferromagnetism in a multiparticle ground state that Mielke [5] has proven to exist in the Hubbard 
model when the single-particle ground state is $N_{\mathrm{d}}$-fold degenerate for filling less than $N_{\mathrm{d}}$.

Finally, a proof of the existence of ferromagnetic ground states for the Hubbard model on a complete graph has been given recently: for one electron added to the half-filled configuration the ground state is maximally ferromagnetic, while for larger fillings the ground state is ferromagnetic but degenerate with respect to the total spin $S[6]$.

In this paper we study the possible occurrence of ferromagnetism in the Hubbard model on a ring by means of a previously developed [7] exact diagonalization procedure, based on the simultaneous application of the translational invariance and of the two distinct spin and pseudospin $S U(2)$ symmetries. We investigate the spin properties of the ground state for chains up to 8 sites for any filling and in configurations not covered by the general results previously mentioned.

\section{Model}

The starting point is the Hubbard Hamiltonian on a ring

$$
H=-t \sum_{i, \sigma}\left(c_{i \sigma}^{\dagger} c_{i+1, \sigma}+\text { h.c. }\right)+U \sum_{i} n_{i \uparrow} n_{i \downarrow}
$$

where we assume $c_{N+1, \sigma}=c_{1 \sigma}$. Here and hereafter $N$ and $N_{\mathrm{e}}$ denote the number of sites and the number of electrons, respectively. It is well-known that this Hamiltonian has two independent $S U(2)$ symmetries in spin and pseudospin space [10], which involve the spin and the charge degrees of freedom, respectively. The first one, which reflects the invariance of $H$ under spin rotation, is the ordinary $S U(2)$ symmetry in spin space, characterized by the generators

$$
S_{z}=\frac{1}{2} \sum_{i}\left(n_{i \uparrow}-n_{i \downarrow}\right), \quad S_{+}=\sum_{i} c_{i \uparrow}^{\dagger} c_{i \downarrow}, \quad S_{-}=\sum_{i} c_{i \downarrow}^{\dagger} c_{i \uparrow} .
$$

The second $S U(2)$ symmetry can be generated by a particle-hole transformation involving only one kind of spin, say $c_{i \downarrow} \rightarrow(-)^{i} c_{i \downarrow}^{\dagger}$, which maps at half filling the repulsive- $U$ Hubbard model into the attractive- $U$ one. The fact that this latter model also shows rotational symmetry in its spin space implies that the Hamiltonian (1) is invariant under the action of a second group, that we denote by $\overline{S U}(2)$, whose generators are

$$
J_{z}=\frac{1}{2} \sum_{i}\left(n_{i \uparrow}+n_{i \downarrow}-1\right), \quad J_{+}=\sum_{i}(-)^{i} c_{i \uparrow}^{\dagger} c_{i \downarrow}^{\dagger}, \quad J_{-}=\sum_{i}(-)^{i} c_{i \downarrow} c_{i \uparrow} .
$$

Finally, the Hamiltonian (1) commutes with the translation operator $T$, which in turn commutes with $S^{2}, S_{z}, J^{2}$ and $J_{z}$. This implies that the eigenstates of $H$ can be classified in terms of the eigenvalues of the five above-mentioned operators.

\section{Results and discussion}

Applying the method developed in Ref. [7], we diagonalize the Hamiltonian (1) expressing its eigenstates as linear combinations of vectors of the form $\left|S, S_{z}, J, J_{z}, P\right\rangle$ for $N$ even, and $\left|S, S_{z}, J_{z}, P\right\rangle$ for $N$ odd, where $\mathrm{e}^{\mathrm{i} P}$ is the eigenvalue of $T$ ( $P$ is thus the total momentum). $J$ is not a good quantum number in the case of $N$ odd, because $J^{2}$ commutes with $H$ only on bipartite lattices. 
In the case of one hole in the half-filled configuration, we find that the Nagaoka state is only reached in the 3-site ring, for any positive value of $U$, and in the 4-site ring, for $U / t$ larger than a critical value which is approximately equal to 10. This special behaviour, absent in any other larger ring, is to be seen as deriving from the anomalous one-dimensional nature of the 3- and the 4-site ring: in the 3 -site case the physics is essentially equivalent to that of the Hubbard model with infinite range hopping [8], whereas the 4-site ring represents the only case in which a ring satisfies the connectivity condition in the spin configuration space, as defined in Ref. [3].

When we consider the 6 -site ring, we find that the most favourable case for spin alignment is for $N_{\mathrm{e}}=4$ (or 8) (general results on the 6-site problem have been previously reported by Callaway et al. [9]). In this case the triplet state has an energy lower than the singlet state, giving rise to unsaturated ferromagnetism for all positive $U$. This result and those for different fillings have a qualitative explanation in terms of the occupancy of single-particle levels. At $U=0$ the singlet and the triplet state are degenerate. For every finite value of $U$ this degeneracy is removed because the Coulomb repulsion on the one hand increases the energy of the singlet, but on the other hand has no effect on the triplet state, which is obtained filling with two electrons the lowest energy level and with two aligned spin the doubly degenerate next highest-energy level. This argument, which emphasizes the importance of degeneracy, can be regarded as an application of the spin Hund rule to the cluster, considered as a single structured atom in which, when spin degeneracy is resolved by the formation of multiplets, the energy is minimized in the state with maximum spin.

Referring again to the 6-site ring, for $N_{\mathrm{e}}=3$ (or 9) we have found a ferromagnetic saturated ground state above $U / t \approx 10$. The same kind of behaviour has also been obtained in the case of a 8-site ring. This agrees with the result reported by Herring [11], who has shown that the ground state of three electrons on an $N$-site chain with periodic boundary conditions is always ferromagnetic, if the interaction potential is symmetric and tends to infinite as the distance between the particles goes to zero. All the results concerning the 6 -site ring and the 8 -site ring are summarized in Table, where the ground state is specified in terms of the quantum numbers $S$ and $P$. For the two geometries considered here $P$ always takes the values $0,2 \pi / N$ and $4 \pi / N$. The changes in the momentum found both for the hexagon and the octagon in the case of odd $N_{\mathrm{e}}$ can be explained referring to known exact results obtained in the two limits $U=0$ and $U=\infty$. For $U=0$ the model becomes trivial and it is immediate to verify that the ground-state values of $P$ are non-vanishing and equal to those reported in Table for small $U / t$. On the other hand, Bethe-ansatz exact results [12] indicate that for infinite $U$ the $P=0$ state is at any filling well separated in energy by the $P \neq 0$ states, thus implying that there must exist a finite value of $U$ at which the ground-state value of $P$ turns to zero. Moreover, we can see that the magnetic behaviour in the two geometries is essentially the same, apart from the case of one hole in the half-filled configuration, where unsaturated ferromagnetism appears only in the 8-site ring, as a consequence of the different degree of degeneracy characterizing the ground states at infinite $U$. 
TABLE

Spin and total momentum of the ground state for the hexagon and the octagon as functions of the occupation number and $U / t$.

\begin{tabular}{l|l|l}
\hline \hline & Hexagon & \multicolumn{1}{|c}{ Octagon } \\
\hline$N_{\mathrm{e}}=2$ & $(S, P)=(0,0) \forall \frac{U}{t}$ & $(S, P)=(0,0) \forall \frac{U}{t}$ \\
\hline$N_{\mathrm{e}}=3$ & $(S, P)=\left(\frac{1}{2}, \frac{\pi}{3}\right) \frac{U}{t}<10$ & $(S, P)=\left(\frac{1}{2}, \frac{\pi}{4}\right) \frac{U}{t}<8$ \\
& $(S, P)=\left(\frac{3}{2}, 0\right) \frac{U}{t}>10$ & $(S, P)=\left(\frac{3}{2}, 0\right) \frac{U}{t}>8$ \\
\hline$N_{\mathrm{e}}=4$ & $(S, P)=(1,0) \forall \frac{U}{t}$ & $(S, P)=(1,0) \forall \frac{U}{t}$ \\
\hline \multirow{2}{*}{$N_{\mathrm{e}}=5$} & $(S, P)=\left(\frac{1}{2}, \frac{\pi}{3}\right) \frac{U}{t}<100$ & $(S, P)=\left(\frac{1}{2}, \frac{\pi}{4}\right) \frac{U}{t}<100$ \\
& $(S, P)=\left(\frac{1}{2}, 0\right) \frac{U}{t}>100$ & $(S, P)=\left(\frac{1}{2}, 0\right) \frac{U}{t}>100$ \\
\hline$N_{\mathrm{e}}=6$ & $(S, P)=(0,0) \forall \frac{U}{t}$ & $(S, P)=(0,0) \forall \frac{U}{t}$ \\
\hline \multirow{2}{*}{$N_{\mathrm{e}}=7$} & $(S, P)=\left(\frac{1}{2}, \frac{\pi}{3}\right) \frac{U}{t}<100$ & $(S, P)=\left(\frac{1}{2}, \frac{\pi}{2}\right) \frac{U}{t}<100$ \\
& $(S, P)=\left(\frac{1}{2}, 0\right) \frac{U}{t}>100$ & $(S, P)=\left(\frac{3}{2}, 0\right) \frac{U}{t}>100$ \\
\hline$N_{\mathrm{e}}=8$ & $(S, P)=(1,0) \forall \frac{U}{t}$ & $(S, P)=(0, \pi) \forall \frac{U}{t}$
\end{tabular}

Finally, we find that for $N=5,7$ in half filling the ground state shows unsaturated ferromagnetism above $U / t \approx 2$. This result does not contradict the Lieb theorem since in this case the lattice is not bipartite.

The extension of this kind of analysis to larger rings and to bidimensional clusters will be the subject of a forthcoming paper.

\section{References}

[1] E.H. Lieb, D.C. Mattis, Phys. Rev. 125, 164 (1962); E.H. Lieb, D.C. Mattis, J. Math. Phys. 34, 891 (1963).

[2] M. Aizenman, E.H. Lieb, Phys. Rev. Lett. 65, 1470 (1990).

[3] Y. Nagaoka, Phys. Rev. 147, 392 (1966); H. Tasaki, Phys. Rev. B 40, 9192 (1989).

[4] E.H. Lieb, Phys. Rev. Lett. 62, 1201 (1989).

[5] A. Mielke, J. Phys. A 24, L73 (1991); Phys. Lett. A 174, 443 (1993).

[6] M. Salerno, Z. Phys. B, to be published; P. Pieri, preprint available on http://xxx.lanl.gov/(cond-mat/9605156).

[7] C. Noce, M. Cuoco, submitted to Phys. Rev. B.

[8] P. van Dongen, D. Vollhardt, Phys. Rev. B 40, 7252 (1989).

[9] J. Callaway, D.P. Chen, R. Tang, Phys. Rev. B 35, 8723 (1987).

[10] C.N. Yang, S.C. Zhang, Mod. Phys. Lett. 34, 759 (1990); C.N. Yang, Phys. Lett. A 161, 292 (1991); M. Pernici, Europhys. Lett. 12, 75 (1990).

[11] C. Herring, Phys. Rev. B 11, 2056 (1975).

[12] B. Doucot, X.G. Wen, Phys. Rev. B 40, 2719 (1989). 